\title{
Molecular-level soil organic matter composition under impervious surfaces
}

YINGHUI WANG ${ }^{1,2}$, YAMENG SHI $^{1}$, GUODONG SUN ${ }^{3}$, JiNTAO Li ${ }^{1,2}$, HUAN CHEN ${ }^{4}$, ALEX T. CHOW ${ }^{4}$, HAMED MAJIDZADEH $^{5}$, JUN-JIAN WANG ${ }^{1}$

${ }^{1}$ School of Environmental Science and Engineering, Southern University of Science and Technology, Shenzhen 518055, China (Correspondence: wangjj@sustech.edu.cn)

${ }^{2}$ School of Water Resources and Hydropower Engineering, Wuhan University, Wuhan 430072, China

${ }^{3}$ College of Geography and Environmental Science, Northwest Normal University, Lanzhou 730070, China

${ }^{4}$ Biogeochemistry \& Environmental Quality Research Group, Clemson University, Clemson, South Carolina 29442, USA

${ }^{5}$ Department of Sciences, Southern New Hampshire University, Manchester, New Hampshire 03101, USA

As we have entered the Anthropocene, the cycling of soil organic matter (SOM) has been increasingly impacted by land-use changes. Understanding the biogeochemical cycling of SOM beneath impervious surface areas (ISA) is essential to assess the future soil functions with global urbanization. However, the impact of ISA on the molecular-level SOM composition remains unknown. Here, we use ${ }^{13} \mathrm{C}$-nuclear magnetic resonance (NMR) and biomarker analyses to elucidate the alteration in SOM composition beneath ISA in a field-controlled study and beneath two real home constructions. The nearby open surface and subsurface lawn soils were used as different references to exclude the impact of the common topsoil removal before soil sealing. The carbon storage under ISA decreases significantly compared to surface lawn soils but did not when compared to subsurface lawn soils. However, the SOM composition under ISA revealed by ${ }^{13} \mathrm{C}-\mathrm{NMR}$ was consistent compared to both references. The chemical composition of SOM exhibited great variation between soils beneath concrete slabs and simulated home construction on a crawl space. Loss in labile compounds like carbohydrate was the major contributor to the degradation of SOM under concrete, while lignin can be additionally consumed under homes built on crawl spaces. With longer-term (up to 114 years) of real home construction on crawl space, SOM lost more carboxyl and carbonyl C and became highly depleted in carbohydrates but enriched in aromatics. This study highlights the significant alteration in SOM chemistry with ISA construction under the context of global urbanization. 